\title{
Application of Parallel Mechanism in Varistructured Quadruped/Biped Human-carrying Walking Chair Robot
}

\author{
Ling-Feng Sang ${ }^{1}$ Hong-Bo Wang ${ }^{1,2}$ Dian-Fan Zhang ${ }^{3}$ Zhen-Hua Tian ${ }^{1}$ Fu-Hai Deng ${ }^{1}$ De-Lei Fang ${ }^{1}$ \\ ${ }^{1}$ Parallel Robot and Mechatronic System Laboratory of Hebei Province, Yanshan University, Qinhuangdao 066004, China \\ ${ }^{2}$ Key Laboratory of Advanced Forging \& Stamping Technology and Science of Ministry of Education, Yanshan University, \\ Qinhuangdao 066004, China \\ ${ }^{3}$ Science and Technology Research Institute, Yanshan University, Qinhuangdao 066004, China
}

\begin{abstract}
For the existing problems of walking chair robot such as simple function, lower bearing capacity and not walking in complex environment, a novel varistructured quadruped / biped human-carrying walking chair robot is proposed. The proposed robot could be used as biped and quadruped walking chair robots. Considering the conversion of the walking chair robot from the quadruped to the biped or vice versa, 6-UPS and 2-UPS+UP (U, P and S are universal joint, the prismatic pair, and sphere joint, respectively) parallel mechanisms are selected as the leg mechanism of the biped walking robot and quadruped walking robot, respectively. Combining the screw theory and theory of mechanism, the degrees of freedom of the leg mechanism and the body mechanism in different motion states are computed so as to meet the requirements of mechanism design. The motion characteristics of the 2-UPS+UP parallel mechanism which is the key part of the walking chair robot are analyzed. Then, the workspace of the moving platform is drawn and the effect of the structural parameters on the workspace volume is studied. Finally, it is found that the volume of the workspace of the moving platform is bigger when the side length ratio and the vertex angle ratio of the fixed platform and the moving platform which are isosceles triangles are close to 1 . This study provides a theoretical foundation for the prototype development.
\end{abstract}

Keywords: Human-carrying walking chair robot, parallel leg mechanism, degree of freedom, varistructure, workspace, kinematics.

\section{Introduction}

It is estimated that more than one billion people around the world (about $15 \%$ of the world's population) have some physical or cognitive disabilities and many of them live in developing countries. Meanwhile, the number of elderly people is increasing. So more people need wheelchairs or other kinds of equipments to assist them to walk. Walking aids for the elderly and the disabled, as its name suggests, are used to help the disabled and the elderly walk freely in complex environment.

Walking aids mainly include wheeled robots and legged human-carrying walking chair robots. The existing wheeled robots are capable of moving in structured and unstructured environments, going up and down stairs and climbing over obstacles ${ }^{[1,2]}$. However, compared with the legged human-carrying walking chair robots, the requirements for the wheeled robots on road surface are strict ${ }^{[3]}$. A legged human-carrying walking chair robot is easier to pass the obstacles, walk through the sand, swamp and other special road surfaces. The legged human-carrying walking chair robots for the elderly and the disabled could expand their activity space and enable them to walk more freely, both indoors and outdoors.

The leg mechanism, which decides the robot performance, is the key part of a walking chair robot ${ }^{[4]}$. By

\footnotetext{
Manuscript received March 30, 2013; revised August 1, 2013

This work was supported by National Natural Science Foundation of China (No.61075099) and FP7-PEOPLE-2012-IRSES: Marie Curie Action "International Research Staff Exchange Scheme" (No. 318902).
}

observing the motion system of animals (including people) in nature, it is found that skeletal muscles are attached to the bones by tendons and arranged in a parallel way. The structure of leg muscles of the human body is shown in Fig. 1 (a). Fig. 1 (b) presents the parallel connection between each muscle and the bone. This parallel-link way of animal is similar to the parallel mechanism (PM), so many scholars tried to use PM as the leg mechanism for walking chair robot. The first biped human-carrying walking robot WL- $15^{[5]}$ with parallel leg mechanism (PLM) was developed by Waseda University in 2001 , and the robots WL-16 $6^{[6]}$ $\mathrm{WL} 16 \mathrm{R}^{[7]}, \mathrm{WL}-16 \mathrm{RII}^{[8]}{ }^{[\mathrm{WL}-16 \mathrm{RIII}}{ }^{[9]}$ and WL-16RIV ${ }^{[10]}$ were proposed later. The mechanism theory for a biped walking robot with PLM was studied by Hebei University of Technology in China ${ }^{[11]}$. The theoretical research on quadruped walking robots with PLM was conducted in [12]. The hexapod walking robot with 2-UPS/UP and (U+UPR) $\mathrm{P}+\mathrm{UP}$ PM as the leg mechanisms were studied in [13-16]. Based on the 3-RPC PM, a walking robot that can move in any directions without adjusting the body posture was designed in [17]. PM has great stiffness, stable structure and no accumulation error. It is easy to solve inverse kinematics and convenient for online real-time calculation by the robot. Therefore, it is a better choice for the leg mechanism of the human-carrying walking chair robot.

At present, the developed walking robots mainly focus on the quadruped and the biped structure. Shigeo Hirose in Tokyo Industrial University and other scholars are engaged in the study of the legged walking robot. They believed that the quadruped walking robot is the best type of the 
legged walking robot, taking into account stability, control and manufacturing cost. So the research on the quadruped robot has social significance and practical value ${ }^{[18]}$. Nevertheless, the biped walking robot has more flexible movement like a man ${ }^{[19]}$. The comparisons of the quadruped walking robot with the biped one are shown in Fig. 2.

When working on an uneven ground, the biped walking robot (right in Fig. 2 (a)) stands on the ground with one leg to maintain balance, which is easy to fall and has poor balance. While the quadruped walking robot (left in Fig. 2 (a)) stands on the ground with three legs, which has good balance, as well as a small moving range of gravity center and high stability. Therefore, the quadruped walking mode is often chosen on the uneven ground.

When walking up and down the stairs, the body inclination of the quadruped walking robot (left in Fig. 2 (b)) is larger than that of the biped walking robot (right in Fig. 2(b)), which makes the rider scared and uncomfortable, and leads to fluctuation of people and gravity center of the body at the same time. So, the biped mode can be chosen when working up and down the stairs.

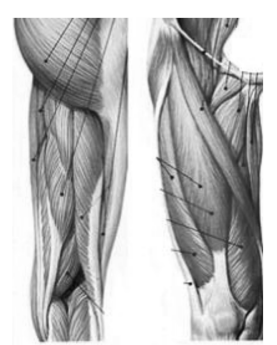

(a) Anatomic assembly

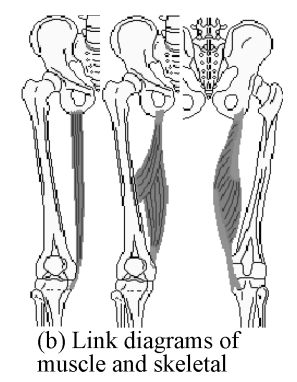

muscle and skeletal
Fig. 1 The human muscle leg system

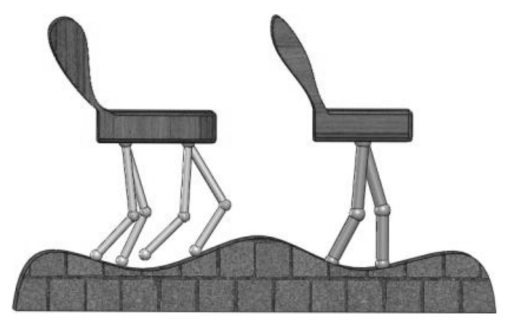

(a) Walking on the uneven ground

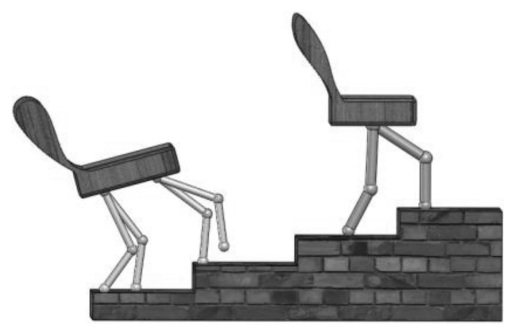

(b) Going up and down stairs

Fig. 2 Compare quadruped robot with biped robot

Now most of the researches on the walking robot mainly focus on the movement structure, function and control method under known environment. But in practical ap- plications, the environment of the most walking robots changes in real time.

In order to make the walking robot adapts to the variable environments and have high carrying capacity, stiffness and stability, a varistructured quadruped / biped humancarrying walking chair robot for the elderly and the disabled is proposed. The proposed robot is a combination of biped walking and quadruped modes. When walking on an uneven ground, the quadrupled mode will be used. When walking up and down the stairs, the biped mode will be adopted. When walking on a flat road, both biped and quadrupled mode can be employed.

\section{Selection of PLM}

In order to meet the walking requirements of a walking chair robot, the body mechanism that consists of an upper platform of PLM and a seat should have 6 degrees of freedom (DOF) when it works in either the quadruped mode or the biped mode. According to the screw theory ${ }^{[20]}$, if the body mechanism has 6 DOF which are linear-independent, then each branch of PLM is not restrict to the body mechanism, i.e., every branch needs 6 kinematics screws of linear independence. As 6-UPS (U, P and S are universal joint, the prismatic pair, and sphere joint, respectively) is a kind of application extremely widespread PM, it is considered as a PLM of the biped walking robot.

When the walking chair robot is transformed from the biped mode to the quadruped mode, the PLM of the walking chair is a PM with three branches. There are 10 kinds of mechanism configurations for transformation from the 6-UPS PM to the PM with three branches, as shown in Table 1.

Table 1 Mechanism configurations for transformation

\begin{tabular}{cccc}
\hline Mechanism & DOF & Mechanism & DOF \\
\hline 3-UPS & 6 & 2-UPS+UPR & 4 \\
2-UPS+SP & 4 & UPS+SPR+SP & 3 \\
2-UPS+SPR & 5 & UPS+UP+UPR & 3 \\
2-UPS+UP & 3 & 3 -RPS & 3 \\
2-UPS+UPU & 5 & 3-UPU & 3 \\
\hline
\end{tabular}

Two factors should be considered for the transformation process:

1) The conversion mechanism should be simple and reliable.

2) Under the premise of meeting requirements of mechanism motion, the walking chair robot does not lead to additional motor number.

Base on the above two points, 10 kinds of mechanism configurations are analyzed as follows:

1) Because the number of DOF is greater than the number of branches, the five mechanisms, including 3-UPS, 2-UPS+SPR, 2-UPS+SP, 2-UPS+UPU and 2-UPS+UPR, need to increase additional motors to make sure the body mechanism has determined the movement, which makes the control more complicated.

2) It is easy for a 3-UPU PM to transform from the quadruped mode to the biped mode or vice veras, but this mechanism cannot be adjusted to random attitude when 
the walking chair robot works in the quadruped mode ${ }^{[21]}$. Each branch of the 3-RPS PM has a constraint force on the moving platform. If 3-RPS PM is selected as the leg mechanism of the walking chair robot, the body mechanism of the walking chair robot may not motion because of the the constraint forces coupling of the multiple branches.

3) In the asymmetric PM with $3 \mathrm{DOF}$, the conversion device of kinematics pair of 2-UPS+UP is simple, and the number of kinematics pairs needed for conversion is less.

4) In order to make sure the walking chair can adjust randomly when moving on the uneven ground, the body mechanism should have 6 DOF. So a PLM of limited DOF needs to increase the degree of freedom to make it not constrained to the body mechanism.

From the above analysis, we can see that the 2-UPS+UP $\mathrm{PM}$ is better to be used as the leg mechanism when the walking robot works in the quadruped mode. Meanwhile, the additional kinematics pair at the end of the PM needs to be added.

\section{Design idea of the whole mechanism}

Based on the modularization idea, the structure of four legs for the robot is the same, and each leg is a modular. The 3-UPS PM is selected as the basic leg mechanism of the walking chair robot, as shown in Fig. 3.

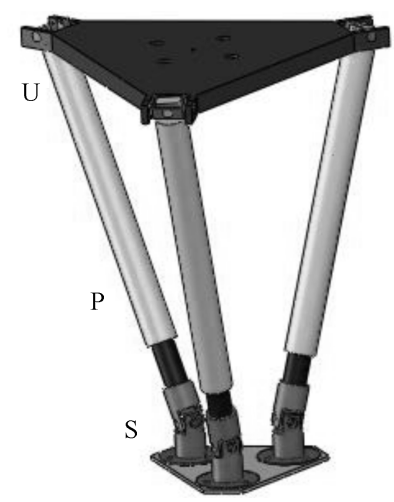

Fig. 3 3-UPS PM

Under the quadruped walking condition, the sphere pair with the moving platform in one branch of the 3-UPS PM is locked, and the PLM is changed into the 2-UPS+UP PM as shown in Fig. 4. Simultaneously, the sphere joint under the lower platform is open. (2-UPS+UP)S hybrid mechanism is formed as four leg mechanisms of the quadruped walking chair robot. When the front foot and rear foot on both sides are combined respectively, the sphere pair with the moving platform is open. Meanwhile, the sphere joint under the lower platform is locked, a biped walking chair robot with 6-UPS PLM is finally formed, as shown in Fig. 5. The locked device is shown in Fig. 6.

\section{DOF of walking chair robots}

\subsection{Single leg mechanism}

The structure of 2-UPS+UP PM is shown in Fig. $7^{[22]}$. The upper and the lower platforms are defined as fixed plat- form and moving platform, respectively, both of which are isosceles triangles. Some structural parameters are defined as $A_{1} A_{2}=A_{1} A_{3}=a, D_{1} D_{2}=D_{1} D_{3}=b, \angle A_{2} A_{1} A_{3}=2 \Phi$, $\angle D_{2} D_{1} D_{3}=2 \phi$. The global coordinate frame $\left\{O_{A}\right\}: O_{A^{-}}$ $X Y Z$ is established at point $A_{1}$ on the fixed platform. Axis $X$ is parallel to $A_{2} A_{3}$. Axis $Z$ is upward and vertical to the fixed platform, and axis $Y$ is determined by the righthand screw rule. The moving coordinate frame $\left\{O_{D}\right\}: O_{D^{-}}$ $X_{D} Y_{D} Z_{D}$ is fixed at point $D_{1}$. Axis $X_{D}$ is parallel to $D_{2} D_{3}$. Axis $Z_{D}$ is upward and vertical to the moving platform, and axis $Y_{D}$ is determined by the right-hand screw rule.

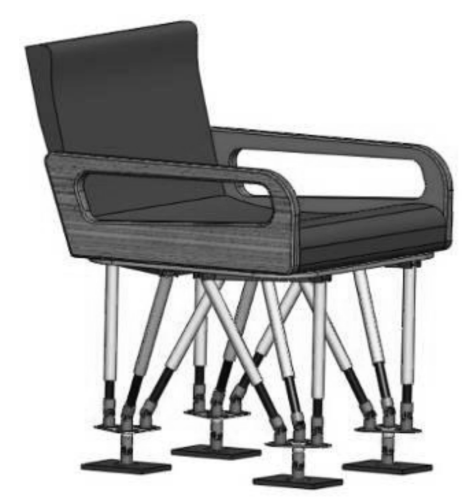

Fig. 4 Quadruped walking chair robot

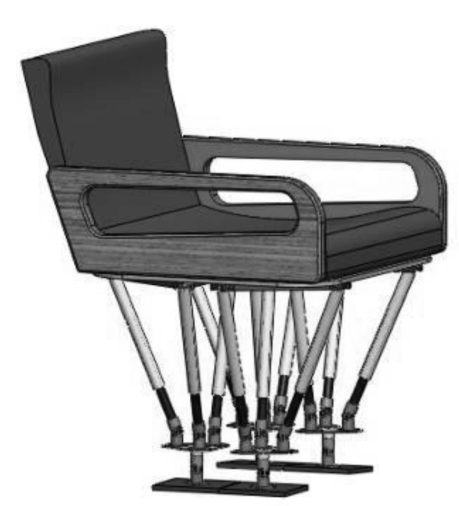

Fig. 5 Biped walking chair robot

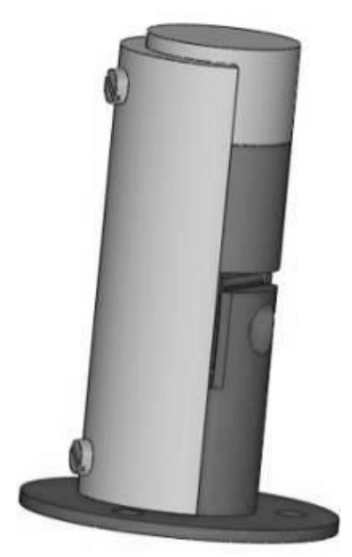

Fig. 6 Locked device 


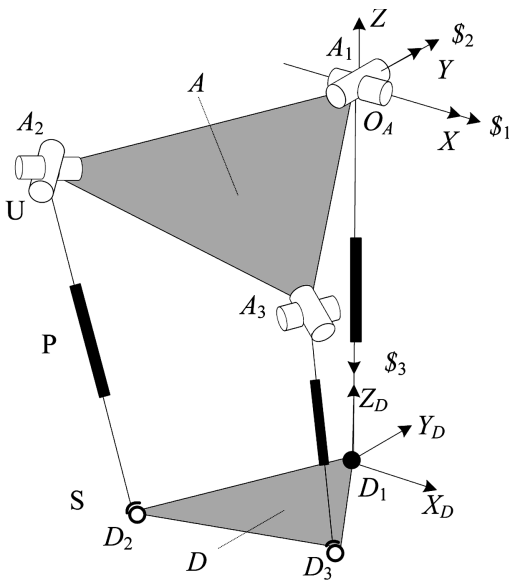

Fig. 7 2-UPS+UP PM

Because the DOF of branch UPS is 6 , and it is not constrained to the moving platform, the DOF of the moving platform is decided by branch UP. The kinematics screws of branch UP are written as

$$
\begin{aligned}
& \$_{1}=\left[\begin{array}{llllll}
1 & 0 & 0 & 0 & 0 & 0
\end{array}\right] \\
& \$_{2}=\left[\begin{array}{llllll}
0 & 1 & 0 & 0 & 0 & 0
\end{array}\right] \\
& \$_{3}=\left[\begin{array}{llllll}
0 & 0 & 0 & 0 & 0 & -1
\end{array}\right] .
\end{aligned}
$$

The constraint screws are solved as

$$
\begin{aligned}
& \$_{1}^{r}=\left[\begin{array}{llllll}
0 & 0 & 0 & 0 & 0 & 1
\end{array}\right] \\
& \$_{2}^{r}=\left[\begin{array}{llllll}
0 & 1 & 0 & 0 & 0 & 0
\end{array}\right] \\
& \$_{3}^{r}=\left[\begin{array}{llllll}
1 & 0 & 0 & 0 & 0 & 0
\end{array}\right] .
\end{aligned}
$$

The constraint screw frame shows that the translation along $X$ and $Y$ directions and the rotation along $Z$ direction are constrained by branch UP. According to $G-K$ formula $^{[20]}$, the DOF of the 2-UPS+UP PM could be obtained by

$$
\begin{aligned}
M= & d(n-g-1)+\sum_{i=1}^{g} f_{i}+\nu= \\
& 6 \times(7-8-1)+15+0=3
\end{aligned}
$$

where $d$ is the order number of mechanism, $n$ is the number of mechanism components, $g$ is the number of kinematics pairs, $f_{i}$ is the number of DOF for the $i$-th kinematics pair, and $v$ is the number of the redundant constraints of the mechanism.

The moving platform has the translational components along axes $X$ and $Y$ which are generated by DOF of 2UPS+UP PM, which leads to the fact that the moving platform does not contact with the ground. Based on walking requirements of the quadruped walking chair robot, a sphere pair is added at the end of the moving platform of 2-UPS+UP PM, and (2-UPS+UP)S hybrid leg mechanism is formed as shown in Fig. 8.

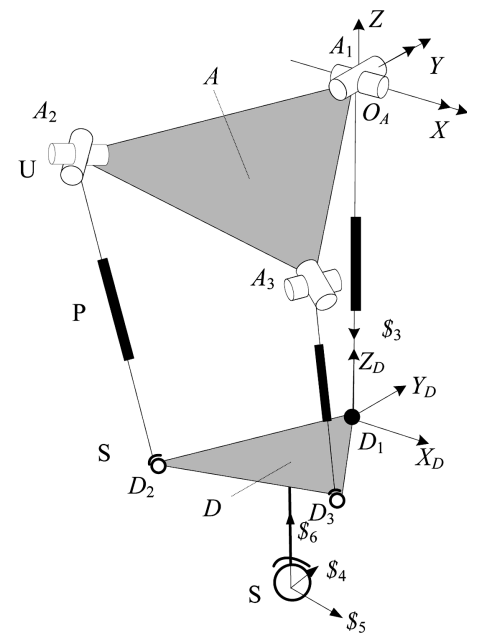

Fig. 8 (2-UPS+UP)S hybrid mechanism

On the basis of $G-K$ formula, the DOF of the hybrid leg mechanism is computed as

$$
\begin{aligned}
M= & d(n-g-1)+\sum_{i=1}^{g} f_{i}+\nu= \\
& 6 \times(8-9-1)+18+0=6 .
\end{aligned}
$$

\subsection{Quadruped walking chair robot}

The quadruped walking robot includes the seat and four (2-UPS+UP)S hybrid mechanisms. The upper platform of each 2-UPS+UP PM is fixed to the seat. Because of the unchanged relative position between the chair and the upper platform of each PM, the seat and the upper platform of 2-UPS+UP PM are regarded as the upper platform of the whole quadruped walking robot. When the leg mechanism of the walking chair robot contacts with the ground, it is defined as the standing leg. When making a step, the leg mechanism is defined as the swing leg. There are three motion states in the motion process of a walking chair robot: The standing with four legs on the ground, the static walking and the dynamic walking. The DOF of each state is analyzed as follows.

1) Standing state with four legs on the ground

The four legs standing is that the four legs mechanism of the quadruped walking chair robot contacts with the ground directly. Under this state, the DOF of the body mechanism of the quadruped walking chair robot is computed as

$$
\begin{aligned}
M= & d(n-g-1)+\sum_{i=1}^{g} f_{i}+\nu= \\
& 6 \times(26-36-1)+72+0=6 .
\end{aligned}
$$

2) Static walking state

Static walking is that one of four legs of the quadruped walking chair robot is the swing leg, and the others are standing legs in the walking process. Under this state, the DOF of the body mechanism of the quadruped walking chair robot is obtained as

$$
\begin{aligned}
M= & d(n-g-1)+\sum_{i=1}^{g} f_{i}+\nu= \\
& 6 \times(20-27-1)+54+0=6 .
\end{aligned}
$$

3) Dynamic walking state 
Dynamic walking is that two legs of the waking chair robot are the standing legs and the others are the swing legs in the walking process. Under this state, the DOF of the body mechanism of the quadruped walking chair robot is solved as

$$
\begin{aligned}
M= & d(n-g-1)+\sum_{i=1}^{g} f_{i}+\nu= \\
& 6 \times(14-18-1)+36=6 .
\end{aligned}
$$

The above analysis of the DOF for the quadruped walking chair robot shows that the mechanism can meet the requirements of quadruped walking.

\subsection{Biped walking chair robot}

Under the biped walking condition, 6-UPS is formed as the leg mechanism of the walking chair. There are two states in the motion process of walking chair robot: Humanlike walking and the standing with the two legs on the ground. The DOF of each state is studied below.

1) Standing with two legs on the ground

The two legs standing is that the two legs of the walking chair robot contact with the ground directly. Under this state, the DOF of the body mechanism of the walking chair robot is

$$
\begin{aligned}
M= & d(n-g-1)+\sum_{i=1}^{g} f_{i}+\nu= \\
& 6 \times(26-36-1)+72+0=6 .
\end{aligned}
$$

2) Human-like walking

Human-like walking is that one leg of the walking chair robot is the swing leg, and the other one is the standing leg. Under this state, the DOF of the body mechanism of the walking chair robot is

$$
\begin{aligned}
M= & d(n-g-1)+\sum_{i=1}^{g} f_{i}+\nu= \\
& 6 \times(14-18-1)+36+0=6 .
\end{aligned}
$$

From the above analysis of DOF for the biped walking chair robot, it is known that the mechanism meets the requirements of the biped walking.

\section{Kinematical characteristics of 2- UPS+UP PM}

\subsection{Position analysis of mechanism}

The 2-UPS+UP PM is the key part of the walking chair robot, so its kinematical characteristics are analyzed. As shown in Fig. 7, the coordinates of each point of the fixed and the moving platforms in the respective coordinate frames could be described as

$$
\begin{aligned}
& A_{1}=\left[\begin{array}{l}
0 \\
0 \\
0
\end{array}\right], A_{2}=\left[\begin{array}{l}
-a s_{\varphi} \\
-a c_{\varphi} \\
0
\end{array}\right], A_{3}=\left[\begin{array}{l}
a s_{\varphi} \\
-a c_{\varphi} \\
0
\end{array}\right], \\
& { }^{D} D_{1}=\left[\begin{array}{l}
0 \\
0 \\
0
\end{array}\right],{ }^{D} D_{2}=\left[\begin{array}{l}
-b s_{\phi} \\
-b c_{\phi} \\
0
\end{array}\right],{ }^{D} D_{3}=\left[\begin{array}{l}
b s_{\phi} \\
-b c_{\phi} \\
0
\end{array}\right]
\end{aligned}
$$

where $s_{\varphi}=\sin (\varphi)$ and $c_{\varphi}=\cos (\phi)$.

${ }^{D} D_{i}$ can be described in the global coordinate frame as

$$
D_{i}={ }_{D}^{A} R^{D} D_{i}+P
$$

where $i=1,2,3,{ }_{D}^{A} R=\left(\begin{array}{lll}x_{l} & y_{l} & z_{l} \\ x_{m} & y_{m} & z_{m} \\ x_{n} & y_{n} & z_{n}\end{array}\right)$ is the rotation transformation matrix from $\left\{O_{D}\right\}$ to $\left\{O_{A}\right\}$, and every column of ${ }_{D}^{A} R$ is direction cosines of the axis $X_{D}, Y_{D}, Z_{D}$ in the globe coordinate frame $\left\{O_{A}\right\}$ respectively, $P$ is the position vector of point $D_{1}$, and $P=\left[X_{o}, Y_{o}, Z_{o}\right]^{\mathrm{T}}$.

The asymmetric 2-UPS+UP PM has geometric constraints as

$$
R_{1} \perp R_{2}, R_{2} \perp l_{1}, R_{2} / / Y_{D}, l_{1} / / Z_{D}
$$

where $R_{1}$ and $R_{2}$ are the first and second revolute axis of the universal joint $\mathrm{U}$ of branch $\mathrm{UP}$, respectively, and $l_{1}$ is the vector of branch UP.

Combining (10) with (12), we get

$$
y_{l}=0, X_{o}=l_{1} z_{l}, Y_{o}=l_{1} z_{m}, Z_{o}=l_{1} z_{n}
$$

The calculation would be simple if we select appropriate Euler angles. Here, $X-Y-X$ Euler angles are selected to express the rotation of the moving platform relative to the global coordinate frame as

$$
{ }_{D}^{A} R=\left[\begin{array}{lll}
c_{\beta} & s_{\beta} s_{\gamma} & s_{\beta} c_{\gamma} \\
s_{\alpha} s_{\beta} & -s_{\alpha} c_{\beta} s_{\gamma}+c_{\alpha} c_{\gamma} & -s_{\alpha} c_{\beta} c_{\gamma}-c_{\alpha} s_{\gamma} \\
-c_{\alpha} s_{\beta} & c_{\alpha} c_{\beta} s_{\gamma}+s_{\alpha} c_{\gamma} & c_{\alpha} c_{\beta} c_{\gamma}-s_{\alpha} s_{\gamma}
\end{array}\right]
$$

where $\alpha, \beta$ and $\gamma$ represent the rotation angle along axis $X$, axis $Y$, axis $X$ in turn in the global frame.

Observing (13), the following equality is obtained as

$$
s_{\beta} s_{\gamma}=0 .
$$

Because of the existing rotation along axis $Y$, we could get

$$
\gamma=0
$$

The rotation transformation matrix ${ }_{D}^{A} R$ could be simplified as

$$
{ }_{D}^{A} R=\left[\begin{array}{lll}
c_{\beta} & 0 & s_{\beta} \\
s_{\alpha} s_{\beta} & c_{\alpha} & -s_{\alpha} c_{\beta} \\
-c_{\alpha} s_{\beta} & s_{\alpha} & c_{\alpha} c_{\beta}
\end{array}\right]
$$

Combining (11) and (17) to (13), we obtain

$$
X_{o}=l_{1} s_{\beta}, Y_{o}=-l_{1} s_{\alpha} c_{\beta}, Z_{o}=l_{1} c_{\alpha} c_{\beta} .
$$


Based on (11) - (17), lengths $l_{2}$ and $l_{3}$ of branches $A_{2} D_{2}$ and $A_{3} D_{3}$ could be derived as

$$
\begin{aligned}
l_{2}^{2}= & l_{1}^{2}+a^{2}+b^{2}+2 a l_{1}\left(s_{\varphi} s_{\beta}-c_{\varphi} s_{\alpha} s_{\beta}\right)- \\
& 2 a b s_{\phi}\left(s_{\varphi} c_{\beta}+c_{\varphi} s_{\alpha} s_{\beta}\right)-2 a b c_{\varphi} c_{\phi} c_{\alpha} \\
l_{3}^{2}= & l_{1}^{2}+a^{2}+b^{2}-2 a l_{1}\left(s_{\varphi} s_{\beta}+c_{\varphi} s_{\alpha} s_{\beta}\right)- \\
& 2 a b s_{\phi}\left(s_{\varphi} c_{\beta}-c_{\varphi} s_{\alpha} s_{\beta}\right)-2 a b c_{\varphi} c_{\phi} c_{\alpha} .
\end{aligned}
$$

\subsection{Workspace analysis of mechanism}

\subsubsection{Constraints of workspace}

The size and shape of the workspace of PM are subject to the following factors.

1) Limitation of the branch length

When the walking chair works, the motion of PM cannot go beyond the range of the branch length, which is

$$
l_{i \min } \leqslant l_{i} \leqslant l_{\text {imax }}
$$

where $l_{i \min }$ is the minimum of the branch length, and $l_{i \max }$ is the maximum of the branch length.

2) Limitation of sphere joint

The rotation scope of sphere joint is constrained by its structure, and the constraint relationship can be described as

$$
\theta_{s i}=\frac{n_{i} \times l_{i}}{l_{i}} \leqslant \theta_{s \max }
$$

where $n_{i}$ is the vector for posture of the sphere joint in the fixed coordinate frame, $l_{i}$ is the branch vector of PM, and $\theta_{\text {smax }}$ is the allowable maximal angle of the sphere joint.

3) Limitation of universal joint

The scope of rotation for a universal joint is expressed by the relationship as

$$
\theta_{u i} \leqslant \theta_{u i \max }
$$

where $\theta_{u i \max }$ is the allowable maximal angle of the universal joint.

4) Interference between the branches

The sizes of the upper and the lower rods of the branch have been defined. The interference problems may exist between the branches in the motion process of mechanism, and the relationship can be written as

$$
d_{i} \geqslant d
$$

where $d_{i}$ is the shortest distance between the branches, $d$ is the maximum diameter of the upper and the lower rods of the branch.

\subsubsection{Workspace of leg mechanism}

Based on the basic design indexes of the walking chair, the structure parameters are selected as $a=300 \mathrm{~mm}, b=$ $100 \mathrm{~mm}, \Phi=\phi=\frac{\pi}{4}$, the ranges of $\theta_{u i}$ and $\theta_{s i}$ are both from $-\frac{\pi}{2}$ to $\frac{\pi}{2}$. The workspace of point $D_{1}$, which is drawn by using the spherical coordinate, is shown in Fig. 9 (a). The inner and outer boundaries of the workspace are described in Fig. 9 (b). Fig. 9 (c) shows a boundary condition of the inner and outer surfaces when $Z$ is in a different height.

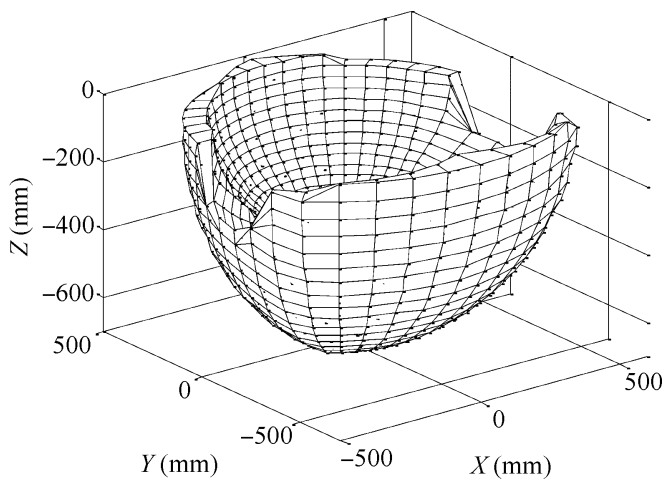

(a) Three-dimensional workspace surface

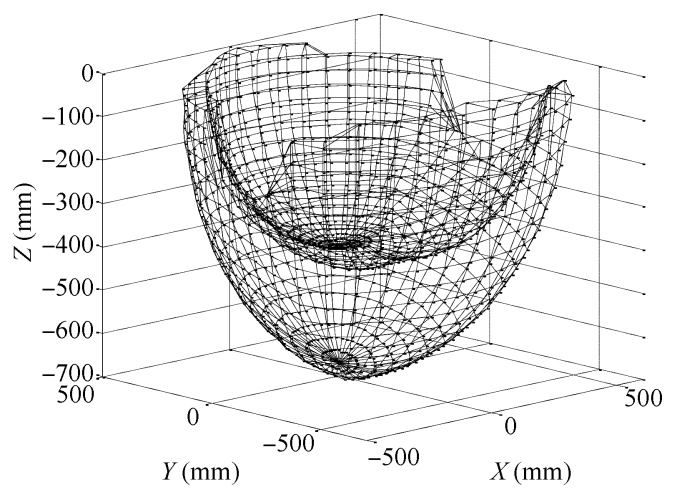

(b) Inner and outer boundaries of workspace

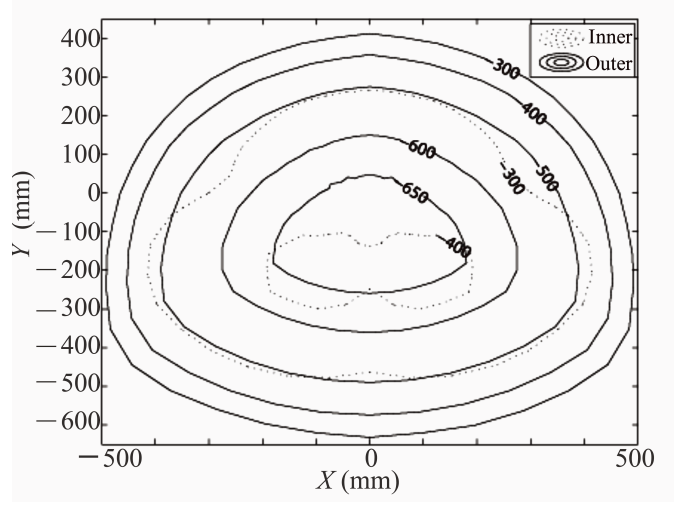

(c) Contour map in $X O Y$ plane

Fig. 9 Workspace of 2-UPS+UP PM

From these figures, it is known that the workspace of point $D_{1}$ of the moving platform is in the inner envelope surface and outer envelope surface. In the range $x=-450 \sim$ $450 \mathrm{~mm}, y=-600 \sim 450 \mathrm{~mm}, z=-400 \sim-700 \mathrm{~mm}$, the workspace has good internal continuity and meets the walking requirements basically. Meanwhile, the workspace gets smaller with increasing height.

\subsection{Effects of parameters on workspace}

The workspace volume, which is selected as the evaluation index to study the effect of parameters on the workspace, is described as

$$
V=f\left(\varphi, \phi, a, b, \theta_{\text {smax }}, \theta_{u \max }, l_{i}\right) .
$$

The workspace volume, which is a multi-parameters 
model, can be solved numerically.

It is obvious that the constraints on the rotation of the mechanism are smaller and the workspace volume is larger when parameters $\theta_{s \max }$ and $\theta_{u \max }$ are larger. Similarly, when the variation range of the branch length is greater, the workspace volume is larger. So we only consider the effect of parameters $\Phi, \phi, a, b$ on the workspace volume.

Let $t=\frac{a}{b}$. Consider combining and driving of leg mechanism, the range of the ratio $t$ is $t>1$. The curve is drawn when $b$ and other parameters are constants, as shown in Fig. 10. It is found that the workspace volume reduces gradually when $t$ increases. Let $k=\frac{\Phi}{\phi}$. When $\phi$ and other parameters are constants, the curve shows that the workspace volume reduces gradually when $k$ increases.

Therefore, when the values of parameters are chosen, angles $\Phi, \phi$ and sides $a, b$ should be as near as possible or the same.

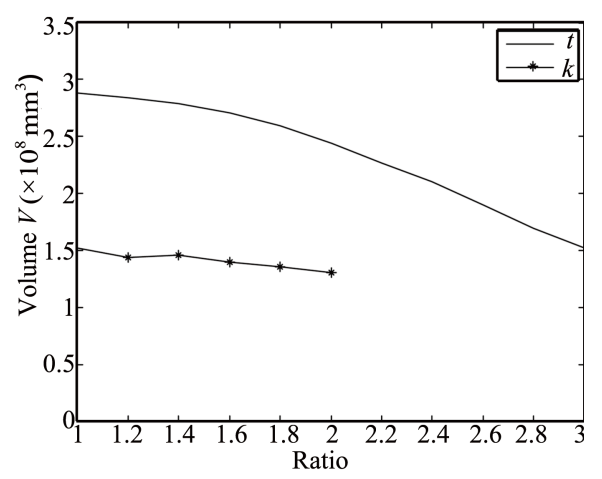

Fig. 10 Effects of parameters on workspace volume

\section{Conclusions}

1) By combing the advantages and disadvantages of the quadruped robot and the biped robot, a novel varistructured quadruped/biped human-carrying walking chair robot is proposed.

2) According to walking requirements, 6-UPS is selected as the leg mechanism of the biped walking chair robot. 2-UPS+UP PM is used as the leg mechanism of the quadruped walking chair robot. Through computing DOF of the leg mechanism and the body mechanism in different motion states, it is verified that the whole walking chair robot meets the requirements of mechanism design.

3) Kinematical characteristics of the 2-UPS+UP PM are analyzed. The result shows that the workspace is of good continuity and suitable for the leg mechanism of the robot. Furthermore, the effects of parameters on the workspace volume are discussed, and the results show that the workspace volume is larger when angles $\Phi$ and $\phi$, sides $a$ and $b$ are as near as possible or the same.

\section{References}

[1] R. Morales, A. Gonzalez, V. Feliu, P. Pintado. Environment adaptation of a new staircase-climbing wheelchair. Autonomous Robots, vol. 23, no. 4, pp. 275-292, 2007.

[2] G. Quaglia, W. Franco, R. Oderio. Wheelchair. q, a motorized wheelchair with stair climbing ability. Mechanism and Machine Theory, vol. 46, no. 11, pp. 1601-1609, 2011.
[3] J. H. Zhang, X. J. Zhang, M. L. Zhang, F. H. Guo. Eight wheel-legged mobile robot platform design and kinematic analysis. Journal of Machine Design, vol. 29, no. 8, pp. 3539, 2012. (in Chinese)

[4] S. C. Fan, S. H. Han, H. R. Gu. The stability of biped walking robot parallel leg mechanism. Journal of Hebei University of Technology, vol. 33, no. 1, pp. 27-31. (in Chinese)

[5] G. Carbone, Y. Sugahara, H. O. Lim, A. Takanishi, M. Ceccarelli. Stiffness performance estimation for biped locomotor WL-15. In Proceedings of IEEE/ASME International Conference on Advanced Intelligent Mechatronics, IEEE, Kobe, Japan, pp. 956-961, 2003.

[6] Y. Sugahara, T. Hosobata, Y. Mikuriya, H. Sunazuka, H. O. Lim, A. Takanishi. Realization of dynamic human-carrying walking by a biped locomotor. In Proceedings of IEEE International Conference on Robotics and Automation, IEEE, New Orleans, Louisiana, USA, pp. 3055-3060, 2004.

[7] Y. Sugahara, Y. Mikuriya, K. Hashimoto, T. Hosobata, H. Sunazuka, M. Kawase, H. O. Lim, A. Takanishi. Walking control method of biped locomotors on inclined plane. In Proceedings of IEEE International Conference on Robotics and Automation, IEEE. Barcelona, Spain, pp. 1977-1982, 2005.

[8] Y. Sugahara, G. Carbone, K. Hashimoto, M. Ceccarelli, H. O. Lim, A. Takanishi. Experimental stiffness measurement of WL-16RII biped walking vehicle during walking operation. Journal of Robotics and Mechatronics, vol. 19, no. 3, pp. 272-280, 2007.

[9] K. Hashimoto, T. Sawato, A. Hayashi, Y. Yoshimura, T. Asano, K. Hattori, Y. Sugahara, H. Lim, A. Takanishi. Static and dynamic disturbance compensation control for a biped walking vehicle. In Proceedings of 2 nd IEEE RAS \& EMBS International Conference on Biomedical Robotics and Biomechatronics, IEEE, Scottsdale, Arizona, USA, pp. 457-462, 2008.

[10] K. Hashimoto, Y. Sugahara, C. Tanaka, A. Ohta, K. Hattori, T. Sawato. Unknown disturbance compensation control for a biped walking vehicle. In Proceedings of IEEE/RSJ International Conference on Intelligent Robots and Systems, IEEE, San Diego, California, USA, pp. 22042209, 2007.

[11] S. C. Fan, S. H. Han, H. R. Gu. The gait planning and trajectory of parallel leg mechanism of biped robot. Machine Design and Research, vol. 20, no.z1, pp. 207-209, 2004. (in Chinese)

[12] H. B. Wang, Z. Y. Qi, Z. W. Hu, Z. Huang. Application of parallel leg mechanisms in quadruped/biped reconfigurable walking robot. Chinese Journal of Mechanical Engineering, vol. 45, no. 8, pp. 24-30, 2009. (in Chinese)

[13] Y. Rong, Z. L. Jin. Dynamic modeling of 3-DOF parallel leg mechanism and peak prediction of servo motor. Optical and Precision Engineering, vol. 20, no. 9, pp. 1974-1982, 2012. (in Chinese)

[14] Y. Rong, Z. L. Jin. Design of parallel mechanical leg of six-legged robot. Optical and Precision Engineering, vol. 20, no. 7, pp. 1532-1541, 2012. (in Chinese)

[15] Y. Rong, Z. L. Jin. Configuration analysis and structure parameter design of six-leg agricultural robot with parallel-leg mechanisms. Transactions of the Chinese Society of Agricultural Engineering, vol. 28, no. 5, pp.9-13, 2012. (in Chinese)

[16] Y. Rong, Z. L. Jin, M. K. Qu. Statics analysis and optimal design of 3-DOF parallel mechanical leg. Transactions of the Chinese Society of Agricultural Engineering, vol. 28, no. 20, pp. 41-49, 2012. (in Chinese)

[17] C. J. Zhang, Y. W. Li. A new walking robot based on 3RPC parallel mechanism. Chinese Journal of Mechanical Engineering, vol. 47, no. 15, pp. 25-30, 2011. (in Chinese) 
[18] S. Hirose, K. Kato. Study on quadruped walking robot in Tokyo Institute of Technology — past, present and future. In Proceedings of IEEE International Conference on Robotics and Automation, IEEE, San Francisco, California, USA, pp. 414-419, 2000.

[19] C. Macro, C. Giuseppe. A new leg design with parallel mechanism architecture. In Proceedings of IEEE/ASME International Conference on Advanced Intelligent Mechatronics, Singapore, pp. 1447-1452, 2009.

[20] Z. Huang, Y. S. Zhao, T. S. Zhao. Advanced Spatial Mechanism. Beijing, China: Higher Education Press, 2006. (in Chinese)

[21] H. B. Wang, L. F. Sang, X. Hu, D. F. Zhang, H. N. Yu. Kinematics and dynamics analysis of a quadruped walking robot with parallel leg mechanism. Chinese Journal of Mechanical Engineering, vol. 26, no. 4, pp. 1-11, 2012.

[22] T. Huang, M. Li, X. M. Zhao ,J. P. Mei, D. G. Chetwynd, and S. J. Hu. Conceptual design and dimensional synthesis for a 3-DOF module of the TriVariant - a novel 5-DOF reconfigurable hybrid robot. IEEE Transactions on Robotics, vol. 21, no. 3, pp. 449-456, 2005.

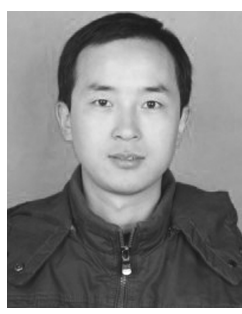

Ling-Feng Sang received his B. Sc. degree in mechanical manufacture and automation from Jiangxi University of Science and Technology, China in 2010. Currently, he is a Ph. D. candidate in College of Mechanical Engineering, Yanshan University, China.

His research interests include parallel mechanism theory and its application.

E-mail: sanglingfeng@163.com

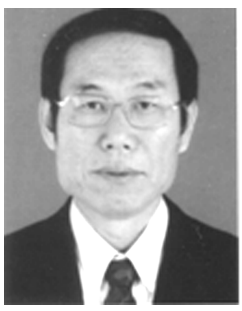

Hong-Bo Wang received his B.Sc. and M.Sc. degrees from Yanshan University, China in 1982 and 1986, respectively. After receiving his $\mathrm{Ph}$. D. degree from Nagasaki University, Japan in 1997, he worked as a deputy general manager in Daihen Corporation. Since 2008, he has become a professor in College of Mechanical Engineering at Yanshan University, China. He has published about 80 journal and conference papers.

His research interests include service robots for the elder and the disabled, medical robot and rehabilitation robot.

E-mail: hongbo_w@ysu.edu.cn (Corresponding author)

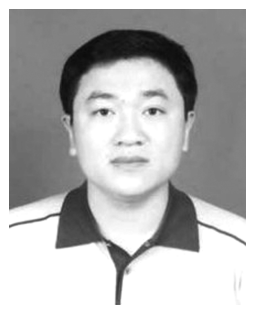

Dian-Fan Zhang received his B.Sc. and M. Sc. degrees in control engineering from Yanshan University, China in 2001 and 2006, respectively, and his Ph.D. degree in mechanical and electronic engineering from Yanshan University, China in 2010. Currently, he works as an asociate research fellow in Science and Technology Research Institute of Yanshan University, China.

His research interests include walking robot engineering, parallel mechanism design and control.

E-mail: zdf@ysu.edu.cn

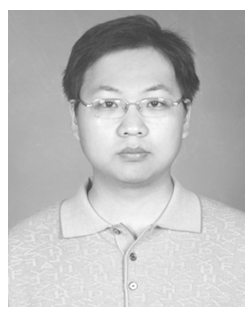

Zhen-Hua Tian received his B. Sc. degree in mechanical engineering from Zhengzhou University, China in 2004. He worked as an engineer in China International Trust and Investment Corporation (CITIC) Heavy Industries Corporation, Ltd, China for 7 years. Currently, he is studying for his M. Sc. degree in College of Mechanical Engineering at Yanshan University, China.

His research interests include robotics, feedback control systems, and control theory.

E-mail: tzhua2011@126.com

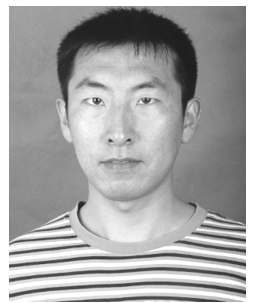

Fu-Hai Deng received his B. Sc. degree in mechanical engineering from Yanshan University, China in 2011. Currently, he is studying for his M. Sc. degree in College of Mechanical Engineering at Yanshan University, China.

His research interests include rehabilitation robot, feedback control systems, and control theory.

E-mail: hongxing8383@126.com.

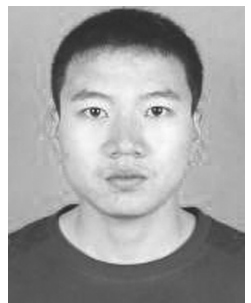

De-Lei Fang received his B. Sc. degree in mechanical engineering from Hebei University of Science and Technology, China in 2011. Currently, he is studying for his M. Sc. degree in College of Mechanical Engineering at Yanshan University, China.

His research interests include robotics, feedback control systems, and control theory.

E-mail: fangdelei365@126.com 